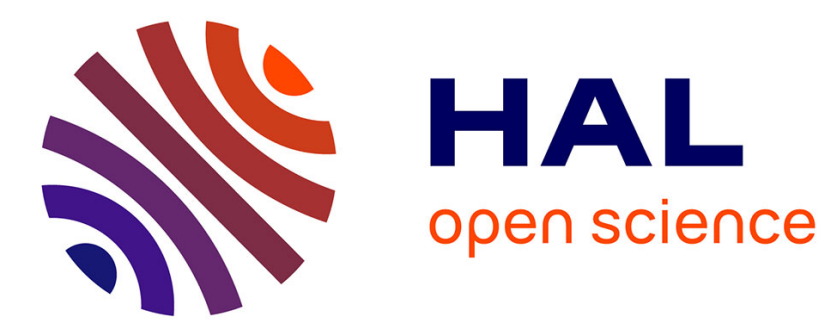

\title{
PROPRIÉTÉS DES JONCTIONS p/n DANS LE SILICIUM OBTENUES PAR IMPLANTATION IONIQUE ET RECUIT RAPIDE
}

\author{
M. Boissy, P. Ruterana, G. Nouet
}

\section{- To cite this version:}

M. Boissy, P. Ruterana, G. Nouet. PROPRIÉtÉS DES JONCTIONS p/n DANS LE SILICIUM OBTENUES PAR IMPLANTATION IONIQUE ET RECUIT RAPIDE. Journal de Physique Colloques, 1983, 44 (C5), pp.C5-401-C5-408. 10.1051/jphyscol:1983558 . jpa-00223143

HAL Id: jpa-00223143

https://hal.science/jpa-00223143

Submitted on 1 Jan 1983

HAL is a multi-disciplinary open access archive for the deposit and dissemination of scientific research documents, whether they are published or not. The documents may come from teaching and research institutions in France or abroad, or from public or private research centers.
L'archive ouverte pluridisciplinaire HAL, est destinée au dépôt et à la diffusion de documents scientifiques de niveau recherche, publiés ou non, émanant des établissements d'enseignement et de recherche français ou étrangers, des laboratoires publics ou privés. 


\title{
PROPRIÉTÉS DES JONCTIONS P/n DANS LE SILICIUM OBTENUES PAR IMPLANTATION IONIQUE ET RECUIT RAPIDE
}

\author{
M.C. Boissy, P. Ruterana ${ }^{+}$et G. Nouet ${ }^{+}$ \\ RTC Za Radiotechnique Compelec, BP 6025, 14001 Caen, France \\ ${ }^{+}$Laboratoire de Cristallographie, Chimie et Physique des. Solides, LA 251, \\ Université de Caen, 14032 Caen, France.
}

RESUME : Quatre techniques de recuit rapide par faisceau d'énergie ont été évaluées de façon comparative du point de vue de leur application à la recristallisation de couches implantées dans le silicium monocristallin :

- le recuit par faisceau laser YAG pulsé $(0,53 \mu \mathrm{m}$ - $100 \mathrm{~ms})$.

- le recuit par faísceau laser continu $(0,48$ à $0,52 \mu \mathrm{m}-1 \mathrm{~ms})$.

- le recuit isotherme rapide par rayonmement infrarouge $\left(1100^{\circ} \mathrm{C}-10 \mathrm{~s}\right)$.

- le recuit par lampe $\left(1100^{\circ} \mathrm{C}-10 \mathrm{~s}\right)$.

\begin{abstract}
INTRODUCTION
La technologie d'élaboration de composants électroniques au silicium tels que les circuits intégrés subnanosecondes, les transistors microondes, les cellules solaires, comprend la rẻalisation d'éléments (base, émetteur) fortement dopés et de très faible épaisseur $(<0,5 \mu \mathrm{m})$. La première étape de la rêalisation de ces êléments consiste en une implantation ionique qui permet par un contrôle prêcis de la dose et de 1 'ërnergie d'implantation, de mấtriser la quantité d'éléments dopants et leur répartition dans le matëriau. Afin de reconstruire le réseau cristallin et d'activer les impuretés dopantes en les faisant migrer vers les sites substitutionnels, on pratique en production un recuit thermique à haute température ( 900 à $\left.1100^{\circ} \mathrm{C}\right)$ pendant un temps assez long (30 mn à quelques heures). Les impuretés implantées sont alors redistribuées ; la profondeur de jonction et le profil de dopage ainsi obtenus dépendent de la durée de ce traitement thermique. De plus la qualité électronique du matériau est dégradée.

Pour réduire la durêe du séjour à haute tempêrature et éviter ces ínconvénients, diverses méthodes de "recuit rapide" sont actuellement étudiées (réf. 1-6). Leur principe de base consiste à accélërer la croissance épitaxiale de la couche dégradée par implantation en portant celle-ci à très haute tempërature $\left(\geqslant 1100^{\circ} \mathrm{C}\right)$ pendant un temps très bref, au moyen d'une irradiation laser, d'un faisceau électronique ou tout autre forme d'ênergie.

Dans cet article, on détaillera tout d'abord les conditions expérimentales mises en oeuvre pour notre étude puis on développera les résultats de l'ensemble des analyses cristallographiques et électriques qui seront ensuite discutés de façon à évaluer les potentialités d'application industrielle de ces techniques de recuit.
\end{abstract}

\section{I - CONDITIONS EXPERIMENTALES}

\section{1 - Nature des êchantillons traitês}

Le matêríau de base, auquel nous avons appliqué les traitements d'implantation ionique et de recuit rapide, est du silicium monocristallin obtenu par la méthode de tirage vertical Czochralski, sauf pour un cas oũ nous avons dû utiliser un matériau obtenu par épitaxie en phase vapeur. L'orientation cristalline de ces substrats est soit (100) soit (III) de façon à pouvoir étudier l'influence de ce paramètre sur les propriêtés du matériau recristaliisé par recuit rapide après implantation ; le niveau de dopage de ce matériau de base est modéré, de type $n$ ou de type p $\left(10^{15} a^{\left.10^{16} \mathrm{~cm}^{-3}\right)}\right.$

En ce qui concerne les conditions d'implantation ionique, nous avons utilisé 2 dopants de type n, 1'arsenic et le phophore et un dopant de type p, le bore, qui sont couramment utilisés pour la fabrication des composants au silicium. Les composants bipolaires rapides requièrent la réalisation de transistors pour lesquels les émetteurs sont très fortement dopés et les épaisseurs d'émetteur et de base sont inférieures aumicron. 
Ceci nous a conduit à retenir des doses d'implantation élevées pour l'arsenic et le phosphore $\left(7.10^{15}\right.$ et $\left.5.10^{16} \mathrm{at} / \mathrm{cm}^{2}\right)$, faibles et moyennes pour le bore $\langle 6.1013 \mathrm{et}$ $7.10^{15} \mathrm{at} / \mathrm{cm}^{2}$ ); d autre part, nous avons utilisé à la fois des énergies d'implantation faibles (autour de $10 \mathrm{keV}$ ) de façon à limiter la densité de défauts générés très profondément dans le matériau et des énergies plus élevées (60 et $100 \mathrm{keV}$ ) qui permet: tent d'obtenir, après recuit, la jonction $\mathrm{p} / \mathrm{n}$ à une profondeur suffisante, comprise entre 0,2 et $0,6 \mu \mathrm{m}$, dans le cas des. méthodes de recuit où la recristallisation s'effectue en phase solide sans diffusion des impuretés dopantes.

\section{2 - Conditions expérimentales de recuit \\ 2.1) Recuit pạ fiaisceau 1aser pulsé}

La durée du pulse est de $100 \mathrm{~ns}$ à $0,53 \mu \mathrm{m}$ de longueur d'onde; le mode de fonctionnement TEM $_{O O}$ assure au faisceau une allure pratiquement gaussienne du laser YAG utilisé pour le recuit. Le diamètre du faisceau est compris entre 50 et $150 \mu \mathrm{m}$ sur la surface de 1 'échantillon. Nous avons utilisé les densités d'énergie de 1.5, 1.9, 2.25 et $2.55 \mathrm{~J} / \mathrm{cm}^{2}$ pour le recuit de nos échantillons. Comme le diamètre du faisceau est petit, on obtient le recuit de toute la plaque de silicium de $75 \mathrm{~mm}$ de diamètre, en programmant les conditions de balayage de sorte qu'il y ait un certain recouvrement des impacts du faisceau. Ce paramètre ayant une influence importante sur les propxiêtés de la couche fondue et recristallisée, nous 1 'avons également fait varier : nous avons ainsi donné à la distance entre les centres d'impact les valeurs 120 , 30 et $8 \mu \mathrm{m}$.

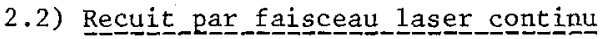

Nous avons utilisê le laser à ion d'Argon à fonctionnement continu, de fabrication Spectra Physics, dont 1 a puissance peut varier entre 0 et $20 \mathrm{~W}$. Le faisceau de $1,5 \mathrm{~mm}$ de diamètre en sortie du laser est focalisé par une lentille convergente sur une dimension de 20 à $100 \mathrm{\mu m}$ de façon à atteindre ainsi des densités de puissance de l'ordre de $10^{5} \mathrm{~W} / \mathrm{cm}^{2}$. Le déplacement du faisceau sur la surface de 1 'échantillon à traiter se fait à I'aide de 2 miroirs galvanométriques. Deux séries d'essais ont été pratiquées à température de substrat élevée :

$$
\text { Puissance }(W) \quad \text { diamètre ( } \boldsymbol{H} \text { m) }
$$

$$
\begin{array}{lrll}
300^{\circ} \mathrm{C} & 10 & \text { à } & 15 \\
350^{\circ} \mathrm{C} & 8 & \text { à } & 9
\end{array}
$$

100 30

\author{
Vitesse $(\mathrm{cm} / \mathrm{s})$ du balayage du \\ faisceau \\ 1 à 10 \\ 12
}

\section{3) Recuit__par_pulse therermique}

Lors du recuit par lampe (HEATPULSE $210 \mathrm{M}$ ) nous avons pu tester 2 conditions de ree cuit que nous avons appliquées respectivement aux échantillons d'orientation cristalline (100) et (111) : le matériaux d'orientation (100) ont été recuits à $1100^{\circ} \mathrm{C}$ pendant $10 \mathrm{sec}$ tandis que ceux d'orientation (111) $1^{\prime}$ 'ont été à $1150^{\circ} \mathrm{C}$ pendant 20 sec. Pour le recuit isotherme rapide (IA-200), tous les échantillons ont été traitês dans les mêmes conditions, à $1200^{\circ} \mathrm{C}$ pendant $10 \mathrm{sec}$.

Ces deux séries d'expériences sont une approche préliminaire de ces nouvelles techniques de recuit; les résultats obtenus serviront de base à des investigations complémentaires destinëes à optimiser ces traitements.

\section{4) Recuit thermique conventionne1}

Pour avoir une base de comparaison, des recuits thermiques conventionnels ont été pratiqués à 700 , et entre 900 et $1000^{\circ} \mathrm{C}$ pendant $20 \mathrm{~min}$, ou une heure, sous $N_{2}$.

II - BILAN DES RESULTATS

1) Etat de surface du materriau et allure de la jonction après recuit

a) Recuit par faisceau laser pulsé

Le système de balayage du laser utilisé est tel que la suite d'impacts du faisceau prêsente un aspect hélicö̈dal sur la surface de la plaque. De ce fait, la distance entre les centres d'impacts n'est pas constante d'une ligne de balayage à I'autre. Aussi pour obtenir un recuit homogène doit-on réaliser un recouvrement tel que la distance entre les centres d'impacts soit toujours inférieure à leur diamètre. Pour les énergies du faisceau de $1.5,1.9$ et $2.25 \mathrm{~J} / \mathrm{cm}^{2}$ nous avons utilisé trois recouvrements en fixant les distances des centres d'impacts à $120 \mu \mathrm{m}, 30 \mu^{\mathrm{m}}$ et $8 \mu^{\mathrm{m}}$. 
Nous analyserons successivement les trois cas :

$120 \mu \mathrm{m}$ : Les impacts du faisceau se détachent les uns des autres dans la direction de déplacement radiale du mouvement du bras laser; ils se recouvrent très partiellement dans la direction tangentielle. C'est dans ce cas que nous avons pu analyser 1 'aspect individuel d'un impact (fig. 1) : l'impact recristallisé se détache du reste du matériau resté amorphe.

$30 \mu \mathrm{m}$ : L'aspect individuel disparâtt; la couche recuite est couverte d'un quadrillage résultant de la superposition des impacts (Fig.2). De plus, la surface est couverte de structures linéaires que d'autres auteurs ont déjà obervées (Réf. 7-10). Elles sont caractërisées par leur direction perpendiculaire à celle du champ électrique du faisceau de recuit et par leur période voisine de la longueur d'onde du faisceau laser pour une incidence normale.

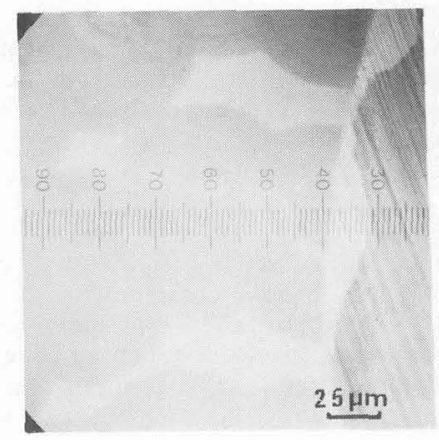

Fig. 1 : Surface et jonction au plus faible recouvrement $d=120 \mu \mathrm{m}$

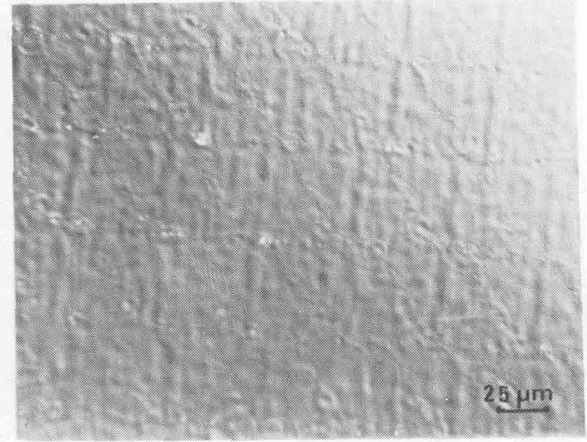

Fig. 2 : Quadrillage en surface $d=30 \mu \mathrm{m}$

$8 \mu \mathrm{m}$ : La surface est uniformément recuite, on retrouve les structures linéaires, de plus, des fissures en surface décorent la plaque. Par endroit, elles se propagent jusqu'en bordure de la plaque. Leur direction est grossièrement normale à celle du balayage tangentiel. La fig. 3 montrge ces défauts qui traversent une zone implantẻe ; leur profondeur est proche de $1000 \AA$. Dès lors, il est évident qu'un recouvrement aussi fort est néfaste pour le matériau recuit. La géométrie de la jonction $\mathrm{p} / \mathrm{n}$ prér sente également des irrégularités. Ainsi, du fait de l'allure gaussienne du faisceau, Il en résulte, que la répartition des impuretés va suivre cette même variation. Pour un faible recouvrement ( $f i g$. 1), la profondeur de jonction peut varier de 0,6 jum à $0,1 \mathrm{um} d u$ centre vers le bord d'un impact, pour une énergie laser de $2,55 \mathrm{~J} / \mathrm{cm}^{2}$. Lorsque le recouvrement augmente, l'écart entre les valeurs extrèmes diminue. Cependant au plus fort recouvrement $(8 \mu \mathrm{m})$, une jonction plane $\mathrm{n}^{\prime e s t}$ toujours pas obtenue

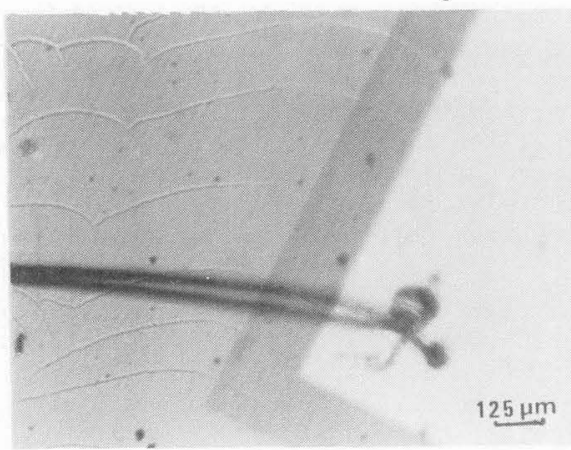

Fig. 3 : Fissures en surface au plus fort recouvrement $d=8 \mu \mathrm{m}$

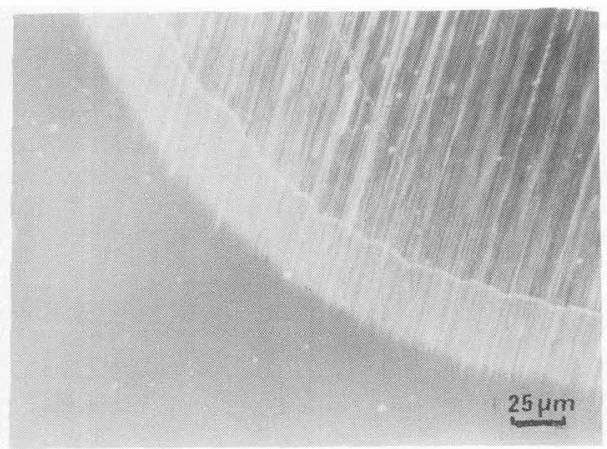

Fig. 4 : Profondeur de jonction au plus fort recouvrement $d=8 \mu \mathrm{m}$

b) Recuit par faisceau laser continu - Lorsque les lignes recuites se recouvrent, la couche présente un aspect homogène de matériau cristallin. Le recouvrement utilisé 
est déterminê au cours d'essais préliminaires en mesurant la largeur des lignes de balayage à puissance ou vitesse variable. On dêtermine une largeur proche de $100 \mathrm{jm}$ en combinant puissance du faisceau et vitesse de balayage. Nous avons fixé la distance entre les centres de lignes à $50 \mu \mathrm{m}$.

c) Recuits par pulse thermique - Lorsque les paramètres (durée, tempẻrature) sont bien ajustés pour la couche à recuire, la surface de la plaque prend l'aspect du silicium cristallin. Cette méthode constitue un excellent moyen pour étudier les mécanismes de la croissance épitaxiale, en phase solide, à haute température.

Pour les recuits par pulse thermique aussi bien que pour les recuits par laser continu, les épitaxies se font en phase solide et compte tenu des durées de recuit, les impuretês restent pratiquement dans le volume d'implantation. Les surfacesde jonction sont parfaitement planes.

2) Structure microscopique des couches après recuit

a) Recuit par faisceau laser pulsé - Le faible diamètre de la zone recristallisëe par impact du faisceau (100 à $120 \mathrm{\mu m})$ nous a donné 1 'occasion d'étudier la structure cristalline d'un impact et son évolution en fonction du recouvrement des impacts. De 1,5 a $2,55 \mathrm{~J} / \mathrm{cm}^{2}$, nous avons mis en évidence, auparavant, que la profondeur de la jonction évolue de 0,2 à $0,6 \mu m$. Ainsi, I'épaisseur de la zone fondue augmente avec $I^{\prime} e ́ n e r-$ gie du faisceau. Le retour en surface du front de fusion s'accompagne de l'épitaxie sur le substrat monocristallin.

L'impact du faisceau laser pulsé se présente sous une forme circulaire ; 1a zone centrale est entièrement recristallisée (fig.5). Lorsque 1 'on se rapproche du bord, la couche devient polycristalline. Les monocristaux y sont orientés au hasard; le diagramme de diffraction se présente sous forme d'anneaux fins caractéristiques du silicium (fig. 6). Au centre du faisceau, la fusion est allée en profondeur dans le matériau monocristallin et 1 'épitaxie, en retour du front de fusion, donne, par conséquent une couche entièrement monocristalline. Sur la përiphérie de I'impact, la zone ne $s^{\prime}$ est pas étendue au delà de la couche amorphe, dès lors, 1 'épitaxie s'est faite sur un matériau de mauvaise qualitê, il en résulte une zone polycristalline, dont les plus gros grains ont de dimensions voisines de $500 \AA$.

Dans le cas du plus faible recouvrement, i1 y a, dans 1a direction tangentie11e, un chevauchement partiel des impacts. On passe alors d'un impact au suivant sans traverser de zone polycristalline... On y trouve des structures linẻaires qui ont la même direction sur toute la zone recuite où elles se trouvent. D'autres auteurs (réf. 10) utilisant un laser $\mathrm{CO}_{2}$ à $10,6 \mu \mathrm{m}$, ont reporté 1 'existence de ces structures. Pour eux, elles sont constituées de zones où le matériau qui reçoit un excès d'énergie s'évapore; leurs observations ettaient faites au microscope optique. Dans notre cas elles sont constituées dans la partie la plus externe de l'impact pax des alternances de zones monocristallines et d'alignements de micro-cristaux ( $f i g .5$ ). Il faut remarquer que ces structures sont localisées sans les zories de recouvrement. Selon la théorie des ondes plasma de surface (Réf. 9), elles devraient être partout sur la surface de 1 'impact; de plus nous avons observé qu'un impact isolé n'en contient pas. En admettant comme font les autres auteurs (Réf. 10), qu'elles proviennent des effets d'interférence entre le faisceau incident et les ondes diffusêes par les impuretés en surface du matériau, on est amené à suggérer, pour expliquer nos observations que les centres diffuseurs sont créês par un premier impact du faisceau laser et que le suivant en subit les effets. De plus à $1,5 \mathrm{~J} / \mathrm{cm}^{2}$, les zones recuites au recouvrement le plus faible n'en contiennent pas; $i l$ existerait donc un seuil de formation de ces centres diffuseurs entre 1,5 et $1,9 \mathrm{~J} / \mathrm{cm}^{2}$ pour le 1 aser YAG utilisé. Lorsque $I^{\prime}$ on augmente le recouvrement, les structures linéaires se rëpandent sur toute la zone recuite (fig. 6).

b) Recuit par faisceau laser continu - La recristallisation sous faisceau laser continu se fait en phase solide, la structure cristalline qui en résulte dépend d'une façon critique de la densité de désordré dans la couche après implantation ionique. Les échantilions préparés par cette méthode de recuit se répartissent en 2 catégories: - Couches très fortement perturbées après implantation (dose 5.1016 at $/ \mathrm{cm}^{2}$ ) : Dans nos conditions de recuits (vitesse de balayage du faisceau laser comprise entre 1 et $10 \mathrm{~cm} / \mathrm{sec}$ ) la densité de désordre des couches était telle que l'épitaxie en phase solide n'a pas été complète. Les couches obtenues sont polycristallines ; cependant, les diagrammes de diffraction ne sont plus formés d'anneaux comme dans le cas du recuit par faisceau pulsé (fig.7). La couche contient deux composantes principales : 
une matrice de croissance Épitaxiale et deux séries de microcristaux dont les directions (111) font un angle de $20^{\circ}$ (fig. 7 a et b).
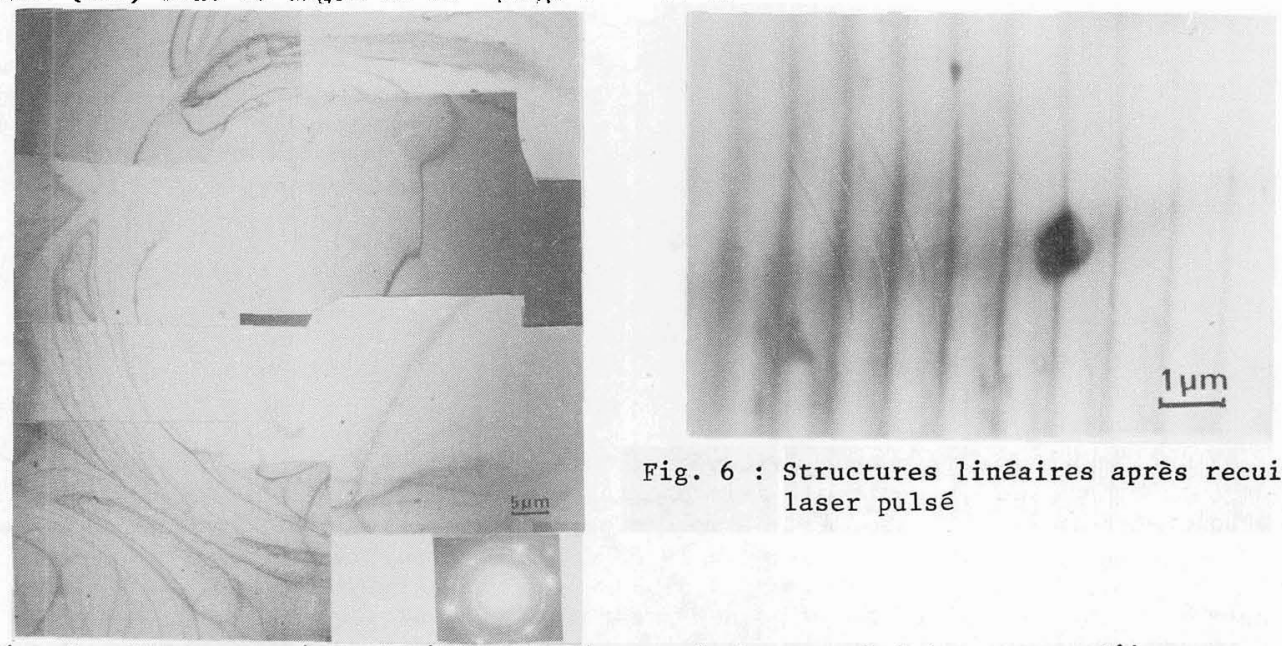

Fig. 6 : Structures linéaires après recuit laser pulsé

Fig. 5 : Structure microscopique d'un impact du laser pulsé, 1 : centre d'impact, 2 : bord de 1'impact.

a)

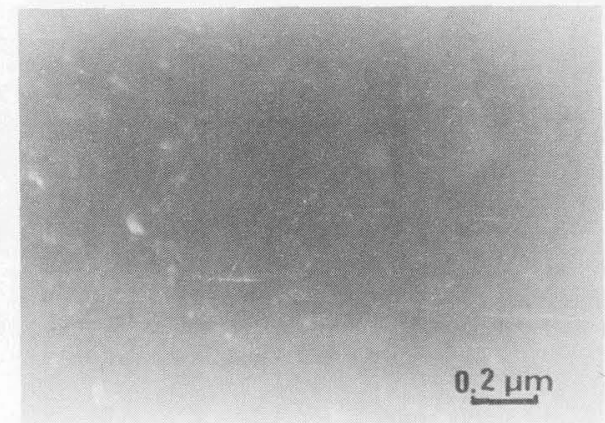

b)

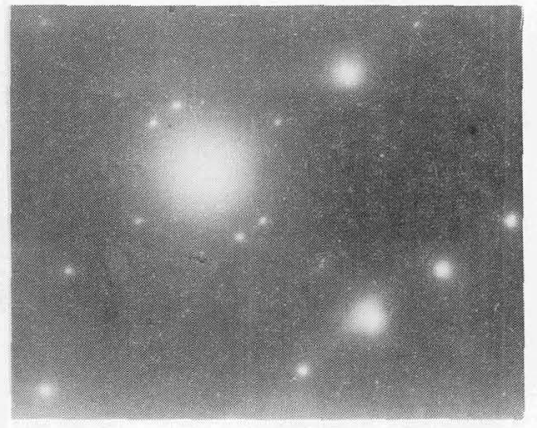

Fig. 7 : Structure microscopique après recuit laser continu, très forte dose a) : champ noir b) : clichê de diffraction.

La formation de ces micro-cristaux peut être expliquée par le processus de nucléation: lorsque la température d'une couche fortement désordonnée s'élève suffisamment, la croissance épitaxiale s'amorce et progresse vers la surface, de plus en plus difficilement, à cause de la densité importante des défauts. En même temps, comme la température est plus élevęe en surface, des atomes peuvent s'y associer et atteindre la taille critique (50 A) de germe de nucléation. Dès lors, la croissance des microcristaux entre en compétition avec l'épitaxie et il en découle une superposition de plusieurs structures dans la couche recuite.

- Implantation de $7.10^{15}$ at $/ \mathrm{cm}^{2}$ a $100 \mathrm{keV}:$ les couches obtenues dans ces conditions d'implantation sont moins désordonnées et la croissance épitaxiale s'effectue à une vitesse suffisamment élevée. La couche recristallise donc entièrement et ne comporte pas de dêfauts.

c) Recuit isotherme rapide - Les observations au microscope mettent en évidence des densités de défauts importantes dans les couches après ce type de recuit (Fig. 8). I1 est donc évident que nos essais de recuit thermique rapide n'ont pas dû être menés dans les meilleures conditions.

d) Recuit par lampe - Au cours du recuit, la température de la plaque est uniforme. On peut donc dire que la croissance épitaxiale a lieu à l'équilibre thermique. Les couches amorphes de départ recristallisent intégralement, et nous n'avons pas observé de dêfauts cristallins dans les plaques implantées à des doses $7.10^{15} \mathrm{at} / \mathrm{cm}^{2}$. 


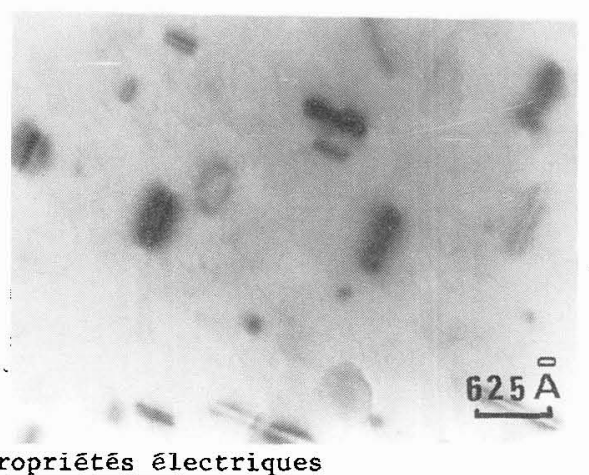
Fig. 8 : Micrographie BF, boucles de dislocation après recuit isotherme rapide.

\section{3) Propriētēs électriques}

a) Activation électrique de la couche recuite - La plus forte activation électrique est obtenue après recuit par faisceau laser pulsé. Ceci est une conséquence des mëcanismes hors d'équilibre, en phase liquide. Les résultats sont comparables pour les deux types de recuit par faisceau laser continu. L'activation électrique semble un peu meilleure pour le traitement à $350^{\circ} \mathrm{C}$, avec une largeur de la ligne recuite égale à $30 \mu \mathrm{m}$. La densité de puissance y est donc plus élevée que dans le recuit à $300^{\circ} \mathrm{C}$, où les lignes recristallisées par balayage ont une largeur proche de $100 \mu \mathrm{m}$. On constate, après recuit par lampe, une conductivité plus élevée dans les couches sur substrats (111) : les conditions de recuit semblent avoir été meilleures que pour les échantillons d'orientation (100). Les bons résultats de conductivité électrique, également obtenus après recuit isotherme rapide ne font pas ressortir que les couches contiennent des défauts cristallins.

b) Caractérisation courant-tension - Recuit par faisceau laser pulsé. L'homogénéité de la couche recuite, l'allure de la surface de jonction jouent un rôle déterminant sur les caractêristiques du dispositif final. Nous avons donc êtudié ces caractéristiques en fonction du recouvrement des impacts du faisceau. Pour les échantillons recuits à $1,9 \mathrm{~J} / \mathrm{cm}^{2}$ après une implantation à forte énergie (100 keV), $1^{\prime}$ épaisseur de la couche dégradée par 1'implantation étant voisine de $0,5 \mu \mathrm{m}$, le désordre est resté important après le recuit au plus faible recouvrement, comme en témoigne la courbe I/V et le facteur d'idéalité de la jonction (n), (fig. 9). Lorsque le recouvrement s'accrô̂t, le désordre résiduel diminue, la caractérisation de la diode s'améliore et on obtient $\mathrm{n}=2$, au plus fort recouvrement.

Autres méthodes de recuit: Les facteurs d'idéalité des diodes obtenues sont compris entre 1,5 et 2 . La qualité électronique est bonne au voisinage des jonctions. Au cours du recuit par faisceau laser continu, l'élévation de la température, au delà de la couche amorphe, peut être suffisante pour que les défauts ponctuels disparaissent. Dans ce cas la zone de charge d'espace est exempte de défauts. Au cours des recuits isotherme rapide et par lampe, tout le volume du matériau est porté à haute température ; les défauts ponctuels, très mobiles, sont les premiers à disparaître.

c) Mesure des longueurs de diffusion - La longueur de diffusion des porteurs minoxitaires dans. Ie volume du matériau est déduite de la mesure de la sensibilité spectrale (fig.10).

Lors du recuit par faisceau laser le traitement thermique $n$ 'affecte pas le volume du matêriau. La qualité du matériau de base n'est donc pas modifiêe par le recuit ; dès lors les longueurs de diffusion des porteurs minoritaires y sont intactes. On obtient des valeurs proches de $150 \mu \mathrm{m}$ qui sont équivalentes à celles présentées par le matériau brut de tirage. Ce résultat en accord avec les caractêristiques I/V, $s$ 'explique par la répartition des impuretés dopantes sur une plus grande profondeur, donc une diminution du dopage et par suite une augmentation de la durée de vie des minoritaires dans la couche superficielie lorsque le recouvrement augmente.

Le recuit par faisceau continu conserve aussi la longueur de diffusion des porteurs minoritaires dans le volume, comme le montre la courbe de sensibilité spectrale dont la pente entre 0,9 et $1,1 \mathrm{\mu m}$ est proche de celle d'un échantillon recuit par faisceau pulsé (Eig. 10)

Par contre, après les recuits par pulse thermique, on constate une réduction notable 
de la longueur de diffusion des minoritaires dans le volume, dans un facteur 2 pour le recuit par lampe et dans un facteur 5 pour le recuit isotherme rapide. Ceci montre donc que, lors de ces recuits thermiques rapides, les contraintes thermiques dégradent la qualité du matériau en volume, tout du moins dans les conditions oû nous les avons mis en oeuvre (figure 10).

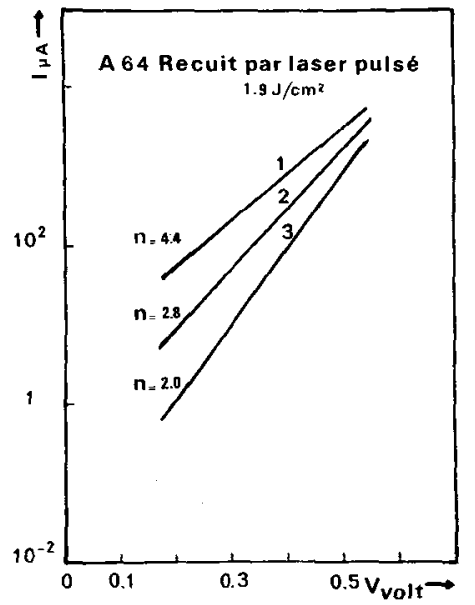

Fig. 9 : Incidence du recouvrement sur les caractéristiques êlectriques

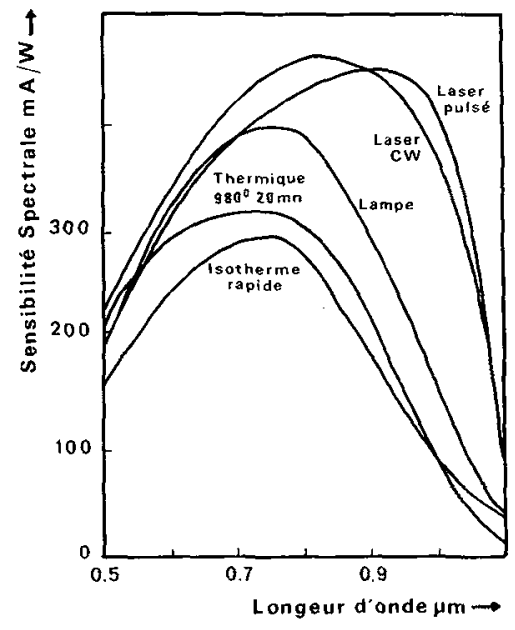

Fig. 10 : Influence du type de recuit sur la réponse spectrale

Conclusion

De nos observations, on peut tirer les conclusions suivantes pour chacune des différentes méthodes de recuit :

- Recuit par faisceau laser pulsé

L'uniformité de la profondeur de jonction est impossible à obtenir, même en utilisant un fort taux de recouvrement des impacts.

Le matériau se dégrade lorsque le recouvrement des impacts du faisceau devient impor-. tant.

L'activation électrique des impuretés implantêes est très efficace.

La longueur de diffusion des porteurs minoritaires dans le substrat est conservẻe.

- Recuit par faisceau laser continu.

La jonction $\mathrm{p} / \mathrm{n}$ est parfaitement plane.

La recristallisation des couches fortement perturbées (doses d'implantation

$\geqslant 5.10^{16}$ at/cn 2) n'est pas obtenue.

$\mathrm{L}$ activation électrique des impuretés est efficace.

La longueur de diffusion des porteurs minoritaires dans le substrat est prêservée.

- Recuit par lampe

Les caractêristiques électriques sont excellentes.

La longueur de diffusion des porteurs minoritaires dans le substrat est réduite de moitié : les plaques sont donc soumises à des contraintes thermiques durant le recuit. L'état crista11in est parfaitement recouvré dans les couches dopées avec des doses moyennes d'ions $\left(7.10^{15} \mathrm{at} / \mathrm{cm}^{2}\right)$.

- Recuit isotherme rapide

Les caractẻristiques électriques sont médiocres.

Les défauts ponctuels subsistent après recuit en densités élevées même pour les doses d'implantation moyennes $\left(7.10^{15} \mathrm{~cm}^{2}\right)$.

La longueur de diffusion des porteurs minoritaires dans le volume du matériau est affectée de façon très sensible (elle est réduite dans un facteur de 1 'ordre de 5), preuve que le matériau a subi des chocs thermiques intenses.

Enfin il faut noter un inconvénient majeur des méthodes de recuit par faisceau laser, provenant de 1 'existence d'effets d'interférences au niveau de l'interface silicium- 
diélectrique, qui ne permet pas d'effectuer ces traitements de recuit par irradiam tion laser sur des plaques comportant en surface des couches d'oxyde ou de nitrure. Ce phênomène n'apparaît pas avec les sources de lumière incohérentes utilisées dans les méthodes de recuit par pulse thermique.

Cette caractéristique, associée aux propriétés énoncées plus haut, conduit à réserver l'utilisation des techniques de recuit par faisceau laser à l'élaboration des cellules solaires dont la technologie ne nécessite pas le dépôt de couches diélectriques et dont les performances sont essentiellement déterminées par la qualité électronique du matériau en volume.

Au contraire, les méthodes de recuit par pulse thermique semblent directement compatibles avec les technologies actuelles de fabrication des transistors micro-ondes et des circuits intégrés. Toutefois il convient de progresser dans l'expérimentation de ces techniques pour en déterminer les conditions optimales de mise en oeuvre de façon à limiter $I$ 'importance des contraintes thermiques.

\section{Remerciements}

Les auteurs tiennent à exprimer leurs remerciements ã J.C. MULLER du Centre de Recherches Nucléaires de Strasbourg et à $G_{\text {. }}$. AUVERT du Centre Norbert Segard du CNET pour l'intérêt qu'ils ont manifestê pour ce travail et la contribution active qu'ils ont apporté. Les auteurs sont également reconnaissants aux Sociétés Arnon Gat Assoc. et Varian de leur avoir donné accès aux équipements de recuit par pulse thermique.

\section{Bibliographie}

1. K. NISHIYAMA, M. ARAI, N. WATANABE, Japanese J.A.P. 19 (1980) 563

2. A. GAT, IEEE - EF Letters, EDL-2, $\mathrm{N}^{\circ} 4$ (1981) 85

3. S. SHATAS, A. GAT, 24th - Electronic Material Conference, Fort Collins, 1982

4. B-Y. TSAUR, J.P. DONNELY, JOHN C.C. FAN, M.W. GEIS, App1. Phys. Lett 39 (1981) 93

5. R.T. FULKS, C.J. RUSSO, P.R. HANLEY, T.I. KAMINS, App1. Phys. Lett. 39 (1981) 604

6. R.T. FULKS, C.J. RUSSO; D.F. DOWNEY, P.R. HANLEY, W.T. STACY, Materials Research Society 1981 Annual Meeting, Boston

7. P.M. FAUCHET, A.E. SIEGMAN, App1. Phys. Lett. 40 (1982) 824

8. N.R. ISENOR, App1. Phys. Latt. 31 (1977) 148

9. S.R.J. BRUECK, D.J. ERLICH, Phys. Rev. Lett. 48 (1982) 1678

10. J.F. YOUNG, J.E. SIPE, J.S. PRESTON, H.M. VAN DRIEL, App1. Phys. Lett. 41 (1982) 261 